\title{
УДК 629.4.066:656.259.13
}

\section{О. И. ЕГОРОВ ${ }^{*}$}

$1^{*}$ Каф. «Электронные вычислительные машины», Днепропетровский национальный университет железнодорожного транспорта имени академика В. Лазаряна, ул. Лазаряна, 2, Днепропетровск, Украина, 49010, тел. +38 (056) 373 15 89, эл. почта egoroffoleg@ukr.net, ORCID 0000-0002-8260-9463

\section{УСОВЕРШЕНСТВОВАНИЕ ПРОЦЕДУРЫ ОПРЕДЕЛЕНИЯ КОЛИЧЕСТВА И ОСНОСТИ ПОДВИЖНЫХ ЕДИНИЦ}

Цель. Построение информационно-управляющих систем, систем контроля движения подвижных единиц, как в пределах станции, так и на прилегающих к ней путях, тесно связано с использованием системами идентификации. В работе рассматривается метод идентификации позволяющий определять количество и осность подвижных единиц, прошедших контрольный участок. Цель работы заключается в усовершенствовании процедуры определения количества и осности подвижных единиц с использованием минимального количества оборудования или без дополнительных затрат, используя уже имеющиеся оборудование установленное на путях для решения других задач. Необходимо предусмотреть возможность повышения достоверности работы других систем идентификации. Методика. Для достижения поставленной цели первоначально был проведен анализ межосевых расстояний подвижных единиц колеи 1520 мм (вагонов и локомотивов). Разработаны математическая и имитационная модели процесса движения подвижных единиц через контрольный участок, с расположенной на нем одной контрольной точкой. Результаты моделирования были обработаны средствами теории вероятности и математической статистики. Результаты. На основании математической модели была разработана имитационная модель, позволяющая проводить эксперименты для разных видов (равноускоренного, равномерного, равнозамедленного) движения подвижных единиц и погрешностей работы датчиков. На основании результатов моделирования были выведены значения коэффициентов времени ожидания для каждого из видов движения, а также универсальный коэффициент для всех видов движения. Проанализированы критичные значения ошибки работы датчика в зависимости от параметров идентифицируемых подвижных единиц. Данный метод критичен к изменению ускорения движения, остановки и реверсивным движениям подвижных единиц. Научная новизна. В данной работе усовершенствован метод идентификации подвижного состава, позволяющий определять количество и осность локомотива и(или) вагонов, прошедших одноточечный контрольный участок. Выполнено усовершенствование процедуры определения количества и осности подвижных единиц путем корректировки коэффициентов времени ожидания фиксации колесных пар. При этом учитываются ошибка работы датчика и значение ускорения движения идентифицируемых подвижных единиц. Практическая значимость. Применение данного метода позволяет повысить достоверность и информативность работы уже разработанных методов идентификации при совместном их использовании. Возможно также применение метода как самостоятельного способа идентификации подвижных единиц с использование минимального количества оборудования.

Ключевые слова: метод идентификации, идентификация подвижного состава, контрольный участок, межосевые расстояния, точечный путевой датчик.

\section{Введение}

Область применения систем различных видов идентификации подвижного состава тесно связана с построением многих информационноуправляющих систем на железнодорожном транспорте. Внедрение подобных систем позволяет решить как главные, так и второстепенные задачи автоматизации технологических процессов широко описанных в работах [1-4]. В ряде случаев применение систем идентификации, как вспомогательных систем, позволяет улучшить качество контроля и управления технологическими процессами на объектах железных дорог и производственных предприятий [57], а также повысить достоверность учета вагонного парка [8].
Широкое распространение получили системы определения статистических характеристик подвижного состава и контроля его перемещения. Эти системы начали разрабатываться и внедряться с момента введения на железных дорогах первых автоматизированных систем учета и управления движением подвижного состава. В основном их задача заключалась в сборе информации на некотором замкнутом пространстве, например на сортировочных станциях. На сегодняшний день именно эти системы являются главными источниками получения информации и управления подвижными единицами на железных дорогах нашей страны и ближнего зарубежья. Системы определения статических характеристик подвижных единиц 
менее критичны с экономической точки зрения по сравнению с системами автоматического определения номера, и их применение является полностью информационно-достаточным для выполнения поставленных задач, а в некоторых случаях и единственно возможным.

К задачам таких систем относится определение следующих статических характеристик подвижного состава, прошедшего контрольный участок: количество осей, количество и осность подвижных единиц, тип подвижных единиц (имеется в виду платформа, крытый вагон и др.) и т. д. При этом, каждая из систем представлена в виде реализованного метода идентификации подвижных единиц, состоящего из алгоритмов сбора, обработки и анализа данных, а также конструктивных особенностей измерительного участка и используемых средств железнодорожной автоматики. В данной работе рассматривается метод определения количества и осности подвижных единиц прошедших одноточечный контрольный участок.

\section{Цель работы}

Известны методы определения количества и осности подвижных единиц с использованием одного датчика на контрольном участке [9]. В общем, идея метода, описанного в [9], заключается в определении момента времени, когда датчик находится между внутренними осями подвижной единицы. Для этого измеряются интервалы времени между наездом на датчик колес подвижной единицы, при этом принимается, что интервалы времени пропорциональны расстоянию между колесами подвижной единицы и обратно пропорциональны скорости движения.

Целью данной работы является усовершенствование приведенного метода идентификации с учетом разных видов движения подвижных единиц и погрешности работы точечного путевого датчика.

\section{Общее описание метода идентификации по- движных единиц}

Метод основан на определении времени, когда контрольная точка находится между внутренними осями подвижной единицы. Отличие этого метода от его аналогов, разработанных ранее, заключается в учете погрешности срабатывания датчика и учете ускорения движения подвижных единиц.

Контрольный участок, на котором происходит идентификация подвижных единиц, состо- ит из следующих устройств железнодорожной автоматики:

- одна контрольная точка, представленная в виде точечного путевого датчика с возможно минимальной зоной срабатывания;

- устройство определения нахождения подвижной единицы на контрольном участке. В качестве этого устройства может быть использована рельсовая цепь или фотодатчик.

Идея метода заключается в следующем: измеряется время $\left(t_{\text {ось }}\right)$ между наездом колес 1-й и 2-й оси подвижной единицы на контрольную точку (расстояние между осями $S_{12}$ ); получен-

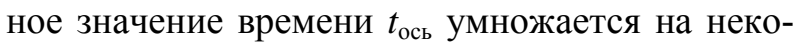
торый коэффициент $\mu$ и получаем время $t_{\text {ож }}$ $\left(t_{\text {ож }}=t_{\text {ось }} \cdot \mu\right)$, в полученном промежутке времени ожидается наезд на контрольную точку следующего колеса подвижной единицы. Далее замеряется следующий интервал между наездом

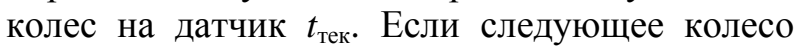
наехало на контрольную точку в течение времени $t_{\text {ож }}\left(t_{\text {тек }}<t_{\text {ож}}\right)$, это значит, что эта ось принадлежит этой же либо спаренной с ней тележке. Если следующее колесо наехало на контрольную точку по истечению времени $t_{\text {ож}}$, это значит, что контрольная точка в этот промежуток времени $\mathrm{t}_{\text {вн }}$ находилась между внутренними осями подвижной единицы. При идентификации 6-ти и более осных подвижных единиц, значение $t_{\text {ож }}$ пересчитывалось. Значение $t_{\text {ось }}$ принималось равным времени между наездом на контрольную точку 3-й и 4-й осями для 8осной подвижной единицы $\left(t_{34}\right.$, расстояние между осями $\left.S_{34}\right), 5$-й и 6-й осями для 12-осных подвижных единиц $\left(t_{56}\right.$, расстояние между осями $\left.S_{56}\right)$ и т.д.

При движении подвижной единицы через контрольную точку подсчитывается количество осей, удовлетворяющих вышеописанному условию для осей, промежуток которых между наездом на контрольную точку меньше $t_{\text {ож}}$. Подсчитанное количество осей, умноженное на 2 (принимаем, что идентификации подлежат подвижные единицы симметричные по осности относительно центра), определяет осность подвижной единицы.

Движение первой и всех последующих осей тележки подвижной единицы (до ее центра) увеличивает значение счетчика осей на 1, а все последующие оси, проходящие контрольную точку, уменьшают его содержимое на 1. Обнуление счетчика осей означает, что подвижная единица полностью прошла через контрольный участок. При этом происходит увеличение счетчика подвижных единиц, прошедших контрольный участок, на 1. 


\section{Математическое описание метода идентификации подвижных единиц}

Были рассмотрены три вида движения отцепа: равноускоренное, равномерное и равнозамедленное.

Погрешность работы датчика характеризуется среднеквадратическим отклонением значения возможной ошибки фиксации центра колеса датчиком $\sigma_{\text {д }}$ распределенного по нормальному закону [10].

Для определения коэффициента $\mu$ были выбраны вагоны и локомотивы разной осности, имеющие минимальное значение расстояния между внутренними осями. Для каждого из видов движения выбирался нижний и верхний интервал значений коэффициента $\mu$. Нижний интервал указывает на значения коэффициента $\mu$, согласно которым ведется подсчет осей, определяющих осность, и вычисляется по формуле

$$
\mu^{\mathrm{H}}=\frac{t_{\text {тек }}}{t_{\text {ось }}} .
$$

Нижний интервал определяется минимальным и максимальным значениями этого коэффициента, для разных видов движения и типов подвижных единиц, удовлетворяющих условию

$$
\begin{aligned}
& \mu_{\min }^{\mathrm{H}}=\operatorname{MIN}\left\{\mu^{\mathrm{H}} \mid t_{\text {ож⿻ }}<t_{\text {ocb }}\right\} \\
& \mu_{\max }^{\mathrm{H}}=\operatorname{MAX}\left\{\mu^{\mathrm{H}} \mid t_{\text {ож⿻ }}<t_{\text {ocb }}\right\}
\end{aligned} .
$$

Верхний интервал указывает на значения коэффициента $\mu$, согласно которым определяется момент расположения датчика между внутренними осями подвижной единицы и вычисляется по формуле

$$
\mu^{\mathrm{B}}=\frac{t_{\mathrm{BH}}}{t_{\mathrm{ocb}}} .
$$

Верхний интервал определяется минимальным и максимальным значениями этого коэффициента, для разных видов движения и типов подвижных единиц, удовлетворяющих условию

$$
\begin{aligned}
& \mu_{\min }^{\mathrm{B}}=\operatorname{MIN}\left\{\mu^{\mathrm{B}} \mid t_{\text {ож⿻ }}>t_{\mathrm{ocb}}\right\} \\
& \mu_{\max }^{\mathrm{B}}=\operatorname{MAX}\left\{\mu^{\mathrm{B}} \mid t_{\text {ож⿻ }}>t_{\mathrm{ocb}}\right\}
\end{aligned} .
$$

Результирующее значение коэффициента $\mu$ находится в диапазоне

$$
\mu \in\left\{\mu_{\text {min }}^{\mathrm{H}}: \mu_{\text {max }}^{\mathrm{H}}\right\} \cap\left\{\mu_{\text {min }}^{\mathrm{B}}: \mu_{\text {max }}^{\mathrm{B}}\right\} .
$$

Для определения значений коэффициента $\mu$ были рассмотрены различные виды движения подвижных единиц с учетом влияния погрешности работы датчика. Максимально возможная погрешность датчика при этом вычислялась по правилу трех сигм и равнялась значению $3 \sigma_{\text {д }}$ [11].

При движении отцепа с положительным ускорением можно сделать однозначный вывод: время между проходом над контрольной точкой 1-ой и 2-й осями подвижной единицы всегда будет больше времени между проходом над точкой последующих осей этой же тележки или спаренной с ней.

При равнозамедленном движении необходимо учитывать тот факт, что время между проходом над датчиком 1-й и 2-й осями подвижной единицы будет меньше времени между проходом над контрольной точкой других осей этой же или спаренной с ней тележки (за исключением 8-осных вагонов, у которых расстояние между 1-2-й и 2-3-й осями тележек равно 1850 мм и 1350 мм соответственно, а также транспортеров), и меньше времени между проходом над датчиком внутренних осей подвижной единицы.

При равномерном движении время между проходом над датчиком 1-й и 2-й осей подвижной единицы больше либо равно времени между проходом над контрольной точкой последующих осей этой же или спаренной тележки, но всегда меньше времени между проходом над контрольной точкой внутренних осей подвижной единицы.

Исходя из этих предположений и воспользовавшись формулами (1) и (3), получаем:

- для равноускоренного движения

$$
\begin{gathered}
\mu^{\mathrm{H}}=\frac{-v_{\text {тек }}+\sqrt{v_{\text {тек }}^{2}-2 \cdot a \cdot\left(S_{\text {тек }}+6 \cdot \sigma_{\text {д }}\right)}}{-v_{1}+\sqrt{v_{1}^{2}-2 \cdot a \cdot\left(S_{12}-6 \cdot \sigma_{\text {д }}\right)}}, \\
\mu^{\mathrm{B}}=\frac{-v_{\mathrm{вH}}+\sqrt{v_{\mathrm{BH}}^{2}-2 \cdot a \cdot\left(S_{\mathrm{BH}}+6 \cdot \sigma_{\text {д }}\right)}}{-v_{\text {ось }}+\sqrt{v_{\mathrm{oсь}}^{2}-2 \cdot a \cdot\left(S_{\mathrm{ось}}-6 \cdot \sigma_{\text {д }}\right)}} ;
\end{gathered}
$$

- для равнозамедленного движения

$$
\begin{gathered}
\mu^{\mathrm{H}}=\frac{-v_{\text {тек }}+\sqrt{v_{\text {тек }}^{2}-2 \cdot a \cdot\left(S_{\text {тек }}-6 \cdot \sigma_{\text {д }}\right)}}{-v_{1}+\sqrt{v_{1}^{2}-2 \cdot a \cdot\left(S_{12}+6 \cdot \sigma_{\text {д }}\right)}}, \\
\mu^{\mathrm{B}}=\frac{-v_{\mathrm{вH}}+\sqrt{v_{\mathrm{BH}}^{2}-2 \cdot a \cdot\left(S_{\mathrm{BH}}-6 \cdot \sigma_{\text {д }}\right)}}{-v_{\text {ось }}+\sqrt{v_{\text {ось }}^{2}-2 \cdot a \cdot\left(S_{\text {ось }}+6 \cdot \sigma_{\text {д }}\right)}} ;
\end{gathered}
$$


- для равномерного движения

$$
\begin{gathered}
\mu^{\mathrm{H}}=\frac{\frac{\left(S_{\text {тек }}-6 \cdot \sigma_{\text {д }}\right)}{v_{1}}}{\frac{\left(S_{12}+6 \cdot \sigma_{\text {д }}\right)}{v_{1}}}=\frac{\left(S_{\text {тек }}-6 \cdot \sigma_{\text {д }}\right)}{\left(S_{12}+6 \cdot \sigma_{\text {д }}\right)}, \\
\mu^{\mathrm{B}}=\frac{\frac{\left(S_{\mathrm{BH}}-6 \cdot \sigma_{\text {д }}\right)}{v_{\text {вн }}}}{\frac{\left(S_{\text {ось }}+6 \cdot \sigma_{\text {д }}\right)}{v_{\text {ось }}}}=\frac{\left(S_{\text {вн }}-6 \cdot \sigma_{\text {д }}\right)}{\left(S_{\text {ось }}+6 \cdot \sigma_{\text {д }}\right)},
\end{gathered}
$$

где $v_{1}$ - начальная скорость наезда на датчик первого колеса подвижной единицы;

$v_{\text {тек }}$ - начальная скорость наезда на датчик последующих колес первой или спаренной с ней тележки;

$v_{\text {ось }}$ - начальная скорость наезда на датчик $n / 2-1$ колеса подвижной единицы (для 6-ти и более осных подвижных единиц);

$v_{\text {вн }}$ - начальная скорость наезда на датчик $n / 2$ колеса подвижной единицы;

$n$ - осность подвижной единицы;

$S_{12}$ - расстояние между 1-ой и 2-ой осями подвижной единицы;

$S_{\text {тек }}-$ расстояние между последними двумя осями проехавшими над датчиком;

$S_{\text {ось }}$ - расстояние между $n / 2-1$ и $n / 2$ осями подвижной единицы (для 6-ти и более осных подвижных единиц);

$S_{\text {вн }}-$ расстояние между внутренними осями подвижной единицы;

$a$ - ускорение движения подвижной единицы;

$\sigma_{\text {д }}-$ среднеквадратическое отклонение погрешности датчика.

После определения допустимых значений коэффициента $\mu$ для любых видов движения, его окончательное значение выбирается как среднее.

\section{Расчет параметров метода идентификации подвижных единиц}

Исходя из описания этого метода, очевидно, что наиболее критичными к процессу идентификации являются подвижные единицы, у которых отношение расстояния между внутренними осями $\left(L_{\mathrm{BH}}\right)$ к расстоянию между 1-й и 2-й осями или осями тележки ближними к центру подвижной единицы, наименьшее. Поэтому при определении границ возможных значений коэффициента $\mu$ были выбраны следующие подвижные единицы, подразделяемые по осности $[12,13]$ :

-4-осный вагон $\left(L_{\mathrm{вн}}=4020\right.$ мМ, $L_{\text {ось }}=$ $=1850 \mathrm{MM})$;
- 6-осный вагон $\left(L_{\text {вн }}=5500\right.$ мМ, $\quad L_{\text {ось }}=$ $=1750 \mathrm{MM})$;

- 6-осный теплово3 $\left(L_{\mathrm{BH}}=4200 \mathrm{MM}, L_{\text {ось }}=\right.$ $=2100 \mathrm{MM})$;

-8-осный вагон $\left(L_{\mathrm{вH}}=2730\right.$ мМ, $L_{\text {ось }}=$ $=1850 \mathrm{MM})$;

-8-осный тепловоз $\left(L_{\mathrm{BH}}=3400 \mathrm{MM}, L_{\mathrm{ocь}}=\right.$ $=2100 \mathrm{MM})$.

При проведении расчетов варьировались следующие параметры:

- начальная скорость - в диапазоне от 0 до $15 \mathrm{M} / \mathrm{c}$;

- ускорение движения - в диапазоне от $-0,3$ до $0,3 \mathrm{M} / \mathrm{c}^{2}$;

- ошибка датчика $\left(\sigma_{\text {д }}\right)$ принималась равной 0,01M.

Результаты вычисления коэффициентов $\mu$ для выбранных подвижных единиц и разных видов движения были сведены в таблицы.

Проанализировав полученные значения коэффициентов $\mu$, были сделаны следующие выводы:

- при равноускоренном движении подвижной единицы необходимо принимать значение коэффициента $\mu$ в диапазоне от 1 до 1,213;

- при равнозамедленном движении подвижной единицы необходимо принимать значение коэффициента $\mu$ в диапазоне от 1,153 до 2,224;

- при равномерном движении подвижной единицы необходимо принимать значение коэффициента $\mu$ в диапазоне от 1 до 1,476.

На основании общего анализа можно сделать следующий вывод: значение коэффициента $\mu$ необходимо принимать из диапазона от 1,153 до 1,213. Разумеется, что наиболее правильно значение коэффициента определяется как среднее значение границ допустимого диапазона, а именно задать его равным 1,183. При этом следует отметить тот факт, что при большем значении начальной скорости движения отцепа зона существования метода идентификации расширяется, а при большем значении ускорения - сужается.

Для апробации полученных значений коэффициентов была разработана имитационная модель.

В процессе моделирования были проведены испытания для 4-х, 6-ти, 8-ми, 12-ти и 20-осных вагонов, локомотивов и транспортеров. Значения скорости и ускорения принимались такими, как и для вычисления коэффициентов $\mu$. Значе-

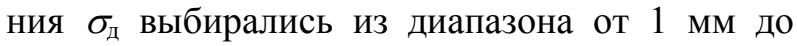
10 мм с шагом 1 мм.

Каждая из исследуемых подвижных единиц проходила 1000 испытаний для каждого из значений скорости, ускорения и среднего квад- 
ратичного отклонения ошибки фиксации оси датчиком. В результате каждая из подвижных единиц проходила контрольный участок 2550000 раз. При этом, ошибки идентификации подвижной единицы были только у 8осного локомотива в 4-х экспериментах при значениях среднеквадратичного отклонения датчика равных 8, 9 и 10 мм, что составляет $1,57 \cdot 10^{-6} \%$ от общего количества экспериментов.

Следует отметить, что в исследованиях принимали участия подвижные единицы, имеющие наиболее критические значения межосевых расстояний для данного метода идентификации.

\section{Научная новизна и практическая значимость}

В работе усовершенствован метод определения количества и осности подвижных единиц с учетом погрешности фиксации колеса датчиком и движения подвижных единиц с ускорением. Проведены исследования и сделаны заключения о влиянии различных факторов на процесс идентификации подвижного состава.

Наиболее удачное практическое применение данной процедуры идентификации возможно в методах определения типа подвижных единиц на трехточечном контрольном участке для фиксации интервала времени нахождения контрольной точки между внутренними осями вагона или локомотива.

\section{Выводы}

Применение одноточечного метода идентификации подвижных единиц наиболее целесообразно как вспомогательного, дополнительного метода при наличии других. Данный метод критичен к параметрам движения (непостоянство ускорения, возможность остановки или изменения направления движения и т.д.) подвижных единиц. Наиболее благоприятные условия для этого метода - размещение контрольного участка на пути движения подвижных единиц, где ускорение движения наиболее постоянно. Применение вычисленного корректирующего коэффициента $\mu$ при использовании одноточечного метода идентификации подвижных единиц, значительно расширяет возможности данного метода и повышает его надежность.

\section{БИБЛИОГРАФИЧЕСКИЙ СПИСОК}

1. Косолапов, А. А. Науковий підхід до створення інтелектуальних транспортних систем: теорія і практика [Препрінт] / А. А. Косолапов // Проблеми економіки та управління на залізничному транспорті ЕКУЗТ 2014. IX Міжнародна науково-практична конференція. - Київ : ДЕТУТ. 2014. - Т. 29, № 2. - С. 45-47.
2. Li, H.-D. Survey of stage plan for railway marshalling station / H.-D. Li, S.-W. He, B.-H. Wang, and Y.-S. Shen // J. of the China Railway Society. 2011. - Vol. 33, № 8. - P. 13-22.

3. Shafia, M. A. Robust train formation planning / M. A. Shafia, S. J. Sadjadi, and A. Jamili // J. of Rail and Rapid Transit. - 2010. - Vol. 224, № 2. - P. 75-90.

4. Косолапов, А. А. Системні характеристики АСК сортувальних станцій / А. А. Косолапов // Інформаційно-керуючі системи на залізничному транспорті. - 2014. - № 4. - С. 47-48.

5. Козаченко, Д. Н. Комплексный анализ железнодорожной инфраструктуры металлургического комбината на основе графоаналитического моделирования / Д. Н. Козаченко, Р. В. Вернигора, Н. И. Березовый // Транспортні системи і технології перевезень : зб. наук. пр. Дніпропетр. нац. ун-ту залізн. трансп. ім. акад. В. Лазаряна - Дніпропетровськ, 2012. - № 4. - С. 55-60.

6. Вернигора, Р .В. Аналіз інтенсивності вантажних поїздопотоків на сортувальних станціях України / Р. В. Вернигора, Л. О. Сльнікова // Транспортні системи і технології перевезень : зб. наук. пр. Дніпропетр. нац. ун-ту залізн. трансп. ім. акад. В. Лазаряна - Дніпропетровськ, 2013. - № 6. - С. 32-35.

7. Лаврухін, О. В. Побудова моделі оптимізації пропуску поїздів на підходах до сортувальної станції / О. В. Лаврухін, П. В. Долгополов, Ю. В. Доценко // Сх.-Свроп. журн. передових технологій. - Харків: 2013. - Вип. 64. - С. 15-17.

8. Долгополов, П. В. Оптимізація роботи залізничних вузлів на основі планування резерву порожніх вагонів / П. В. Долгополов, І. М. Дробот, О. Ю. Рогозін // Зб. наук. праць УкрДАЗТ. - Харків : УкрДАЗТ, 2010. - № 112. - С. 108-112.

9. А. с. № 1039777 СССР, МКИЗ В $61 \mathrm{~L} \mathrm{1/16.}$ Устройство счета вагонов / Ю. В. Соболев, О. С. Попов, Г. И. Загарий, А. И. Синица, П. М. Бобров, В. П. Унтилов, В. Г. Иванов, Э. Н. Суд (СССР). $3401170 / 27-11$; заявл. 24.02.82; опубл. 07.09.83, Бюл. № 33, 4 с.

10. Штанке, А. Э. Исследование и выбор путевых датчиков в системе диспетчерского контроля / А. Э. Штанке, И. Г. Красовская // Совершенствование устройств железнодорожной автоматики и телемеханики : Межвуз. сб. науч. ст. - Свердловск, 1973. - Вып. 34. - С. 59-66.

12. Гмурман В. Е. Теория вероятностей и математическая статистика / В. Е. Гмурман. - Москва : Высшая школа, 1999. - 479 с.

13. Грузовые вагоны колеи 1520 мм железных дорог СССР. Альбом. - Москва : Транспорт, 1989. - 173 с

14. Раков В. А. Локомотивы и моторвагонный подвижной состав железных дорог Советского Союза (1976-1985) / В. А. Раков. - Москва : Транспорт, 1990. - 238 c.

Статья рекомендована к публикации д.т.н., проф. И. В. Жуковицким (Украина)

Поступила в редколлегию 18.11.2015.

Принята к печати 19.11.2015. 


\section{О. Й. ЄГОРОВ}

\section{УДОСКОНАЛЕННЯ ПРОЦЕДУРИ ВИЗНАЧЕННЯ КІЛЬКОСТІ I ОСНОСТЬ РУХОМИХ ОДИНИЦЬ}

Мета. Побудова інформаційно-керуючих систем, систем контролю руху рухомих одиниць, як в межах станції, так і на прилеглих до неї шляхах, тісно пов'язане з використанням системами ідентифікації. У роботі розглядається метод ідентифікації який дозволяє визначити кількість і осность рухомих одиниць, що пройшли контрольний ділянку. Мета роботи полягає в удосконаленні процедури визначення кількості та осності рухомих одиниць 3 використанням мінімальної кількості обладнання або без додаткових витрат, використовуючи вже наявні обладнання встановлене на шляхах для вирішення інших завдань. Необхідно передбачити можливість підвищення достовірності роботи інших систем ідентифікації. Методика. Для досягнення поставленої мети спочатку був проведений аналіз міжосьових відстаней рухомих одиниць колії 1520 мм (вагонів і локомотивів). Розроблено математична і імітаційна моделі процесу руху рухомих одиниць через контрольний ділянку, з розташованою на ньому однієї контрольної точкою. Результати моделювання були оброблені засобами теорії ймовірності та математичної статистики. Результати. На підставі математичної моделі була розроблена імітаційна модель, що дозволяє проводити експерименти для різних видів (рівноприскореного, рівномірного, рівноуповільненого) руху рухомих одиниць і похибок роботи датчиків. На підставі результатів моделювання були виведені значення коефіцієнтів часу очікування для кожного з видів руху, а також універсальний коефіцієнт для всіх видів руху. Проаналізовано критичні значення помилки роботи датчика в залежності від параметрів ідентифікованих рухомих одиниць. Даний метод критичний до зміни прискорення руху, зупинки та реверсивним рухам рухомих одиниць. Наукова новизна. У даній роботі вдосконалено метод ідентифікації рухомого складу, що дозволяє визначати кількість і осность локомотива і (або) вагонів, що пройшли одноточкову контрольну ділянку. Виконано вдосконалення процедури визначення кількості та небезпеки рухомих одиниць шляхом коригування коефіцієнтів часу очікування фіксації колісних пар. При цьому враховуються помилка роботи датчика і значення прискорення руху ідентифікованих рухомих одиниць. Практична значимість. Застосування даного методу дозволяє підвищити достовірність та інформативність роботи вже розроблених методів ідентифікації при спільному їх використанні. Можливо також застосування методу як самостійного способу ідентифікації рухомих одиниць 3 використання мінімальної кількості обладнання.

Ключові слова: метод ідентифікації, ідентифікація рухомого складу, контрольна ділянка, міжосьові відстані, точковий колійний датчик.

\section{O. YEHOROV}

\section{IMPROVEMENT PROCEDURES FOR DETERMINING THE QUANTITY AND NUMBER OF AXLES OF ROLLING UNITS}

Purpose. Building of information and control systems, rolling unit traffic control systems, both within the station and on the adjacent tracks, is closely linked to the identification systems. This paper presents the identification method allowing to determine the quantity and number of axles of rolling units that have passed the control section. The purpose of this work is to improve procedures for determining the quantity and number of axles of rolling units with minimal equipment or no additional cost, using the existing equipment installed on the tracks for other tasks. It should be possible to improve the reliability of other identification systems. Methodology. To achieve this purpose at first the analysis of rolling unit axle spacing for $1520 \mathrm{~mm}$ gauge (of carriages and locomotives) was conducted. There were developed the mathematical and simulation models of rolling unit movement process through the control section, with a single control point located on it. The simulation results were processed by means of the theory of probability and mathematical statistics. Results. On the basis of the mathematical model there was developed the simulation model that allows to carry out experiments for different kinds (uniformly accelerated, uniform, uniformly decelerated) of motion of rolling unit and sensor errors. Based on simulation results there were derived the values of waiting time coefficients for each type of motion, as well as the universal coefficient for all types of motion. The critical sensor error values were analysed depending on the parameters of identified rolling units. This method is critical to the change in acceleration, stop and reverse movements of rolling units. Originality. This paper presents the improved rolling stock identification method that allows to determine the quantity and number of axles of locomotive and (or) carriages, which passed the single-point control section. The procedure for determining the quantity and number of axles of rolling units was improved by adjusting the waiting time coefficients for wheelset fixing. This takes into account the sensor error and the value of the identifiable rolling unit acceleration. Practical value. Application of this method allows to increase the reliability and informative value of already developed identification methods provided their joint use. This method can be also applied as an independent method for rolling unit identification using the minimum equipment.

Keywords: method of identification, rolling stock identification, control section, axle spacing, point track transducer. 